\title{
ESTUDO DE COMORBIDADE: SOFRIMENTO PSÍQUICO E ABUSO DE DROGAS EM PESSOAS EM CENTROS DE TRATAMENTO, MACAÉ - BRASIL
}

\author{
Erotildes Maria Leal ${ }^{1}$, Pedro Gabriel Godinho Delgado², RobertMann ${ }^{3}$, Carol Strike ${ }^{4}$, Bruna Brands ${ }^{5}$, Akwatu Khenti ${ }^{6}$
}

${ }^{1}$ MD, Ph.D. Professora Adjunto da Universidade Federal do Rio de Janeiro - Campus Macaé, Rio de Janeiro, Brasil. E-mail: erotildesleal@macae.ufrj.br

${ }^{2}$ MD, Ph.D. Professor Adjunto do Instituto de Psiquiatria da Universidade Federal do Rio de Janeiro. Rio de Janeiro, Brasil. E-mail: pedrogabrieldelgado@ipub.ufrj.br

${ }^{3}$ Ph.D. Investigator, Centre for Addiction and Mental Health. University of Toronto. Toronto, Canada. E-mail: robert_mann@ camh.net

${ }^{4}$ Ph.D. Investigator. Health Systems Research and Consulting Unit. Centre for Addiction and Mental Health. University of Toronto. Toronto, Canada. E-mail: carol_strike@camh.net

${ }^{5}$ Ph.D. Investigator. Office of Research and Surveillance. Drug Strategy and Controlled Substances Programme. Health Canada and Public Health and Regulatory Policies. Center for Addiction and Mental Health. University of Toronto. Toronto, Canada. E-mail: bruna_brands@camh.net

${ }^{6}$ MSc. Director Office International Health. Assistant Professor at University of Toronto. Dalla Lana School of Public Health. Toronto, Canada. E-mail: akwatu_khenti@camh.net

RESUMO: Estudo da prevalência de sofrimento psíquico entre pessoas em tratamento em um serviço de atenção diário público e dois centros de internação privados, sendo um prestador de serviços ao Sistema Único de Saúde, da cidade de Macaé, Rio de Janeiro, Brasil. Estudo transversal. Foi aplicado questionário para caracterização dos participantes, adaptado do formulário EULAC-CICAD. A escala de Kessler-10 foi utilizada para rastreamento do sofrimento psíquico. Participaram 60 pessoas, sendo 88,3\% homens. Do total, 51,5\% estavam na faixa de 24 a 39 anos de idade. Depressão foi o principal diagnóstico prévio referido em $35 \%$, seguido por ansiedade em $33,33 \%$. Durante o tratamento atual, a ansiedade foi o principal diagnóstico referido em $8,3 \%$, seguido de depressão em $28,3 \%$. Dos entrevistados, $34 \%$ apresentaram níveis severos e 27,2\% muito severos de sofrimento psiquico. Conclui-se que os níveis de sofrimento psíquico severo e muito severo em $61,2 \%$ encontrados com a escala K-10, especialmente sensível para ansiedade e depressão, são semelhantes para esta população nos estudos internacionais.

DESCRITORES: Comorbidade. Sofrimento psíquico. Transtornos relacionados ao uso de substâncias. Assistência em saúde mental.

\section{COMORBIDITY STUDY: PSYCHOLOGICAL DISTRESS AND DRUG ABUSE AMONG PEOPLE IN TREATMENT CENTRES, MACAÉ - BRASIL}

\begin{abstract}
Study of prevalence and psychological distress among people attending a public day care service and two private in patients services in the city of Macaé, Rio de Janeiro, Brazil. Cross-sectional study. A questionnaire adapted by the form EULAC-CICAD was applied to characterize the participants. The scale of Kessler-10 was used as a screening tool for psychological distress. The sample was 60 people: $88.3 \%$ men and $51.5 \%$ between $24-39$ years old. Depression was referred as the most important previous diagnosis in $35 \%$, followed by anxiety in $33.33 \%$. During the current treatment this relationship changed: anxiety was the main diagnosis in $38.3 \%$, followed by depression in $28.3 \%$. The $34 \%$ of the sample had severe psychological distress and $27.2 \%$ very severe psychological distress. The levels of psychological distress severe and very severe in $61.2 \%$ screened by K-10, a scale especially sensitive to anxiety and depression, are similar to international studies on this population.
\end{abstract}

DESCRIPTORS: Comorbidity. Substance use-related disorders. Psychological distress. Mental health assistance.

\section{ESTUDIO DE LA COMORBILIDAD: DISTRES PSICOLÓGICO Y ABUSO DE DROGAS EN PERSONAS EN CENTROS DE TRATAMIENTO, MACAÉ - BRASIL}

\begin{abstract}
RESUMEN: Estudio de prevalencia de distrés psicológicos entre personas que acuden a los servicios públicos de atención de día y dos centros de hospitalización privada en la ciudad de Macaé, Rio de Janeiro, Brasil. Estudio transversal. Se aplicó un cuestionario adaptado de lo formulario de la EULAC-CICAD para caracterizar a los participantes. Para la detección de los distrés psicológicos se utilizó la escala de Kessler-10. Participaron 60 personas, y los hombres eran 88.3\%. De la muestra total, el 51.5\% tenían entre 24 a 39 años de edad. La depresión fue el principal diagnóstico relatado en 35\%, seguido de la ansiedad en 33.33\%. Durante el tratamiento actual la ansiedad fue el diagnóstico principal en $38.3 \%$, seguido de depresión en $28.3 \%$. De los encuestados el $34 \%$ tenían niveles graves de distrés psicológicos y el $27.2 \%$ niveles muy graves. Las conclusiones de que los niveles de distrés psicológico grave y muy grave en $61.2 \%$ identificados pela escala K-10, especialmente sensibles a la ansiedad y la depresión, son similares a los estudios internacionales de esta población.

DESCRIPTORES: Comorbilidad. Distrés psicológico. Trastornos relacionados con sustancias. Atención en salud mental.
\end{abstract}




\section{INTRODUÇÃO}

Os problemas de saúde mental são comuns ${ }^{1-5}$ entre os usuários de substâncias psicoativas. Há um alto risco para o uso prejudicial de álcool e outras drogas em pessoas com problemas mentais e também um risco elevado de ocorrência de problemas mentais entre pessoas em uso prejudicial de drogas. Esta associação afeta significativamente não apenas a qualidade de vida dessas pessoas como cria dificuldades especiais ${ }^{6-11}$ para o tratamento dos problemas que decorrem do uso prejudicial de substâncias psicoativas. Em função disso é importante determinar se os centros de tratamento para os problemas decorrentes de drogas oferecem cuidado para as comorbidades. Se considerarmos que problemas de saúde mental que não preenchem critérios para o diagnóstico de transtornos mentais propriamente ditos têm altíssima prevalência, os riscos decorrentes desta associação ficam ainda maiores. Estudos brasileiros $^{12-13}$ indicam, por exemplo, que sintomas psiquiátricos que não preenchem critérios para o diagnóstico de transtornos mentais têm prevalência de $56 \%$ dentre a demanda das Equipes de Saúde da Família. Sofrimento psíquico é uma das categorias utilizadas para identificar os quadros constituídos por tais sintomas.

Estudos que exploram, separadamente, a prevalência de uso de substâncias psicoativas e a prevalência de transtornos mentais no Brasil não são raros. ${ }^{14-18}$ Dentre os transtornos mentais mais comuns, o transtorno de dependência ao álcool é o mais frequente $(7,6-14,9 \%)$, seguido pela depressão maior (3-13\%), distimia (4,9\%), transtorno bipolar $(0,6 \%-2,1 \%)$, psicoses não-afetivas $(0,3 \%-2,4 \%)$, pânico $(1,3 \%)$ e dependência por drogas ilícitas $(1,0 \%)$.

Os estudos que exploram a associação, em uma mesma pessoa, de transtornos decorrentes do uso de substâncias psicoativas e transtornos mentais, entretanto, são poucos, em especial em cidades brasileiras de médio porte. Embora existam cerca de 60 municípios brasileiros com porte semelhante ao de Macaé, não há relatos de estudos que tenham coberto os serviços ligados ao Sistema Único de Saúde (SUS) de cidade com número similar de habitantes e tenham constituído amostras epidemiologicamente significativas para esse percentual de habitantes. Isto torna evidente a relevância dos estudos que se proponham a considerar esta temática. Principalmente se levarmos em conta que a bibliografia internacional ${ }^{6-11}$ que discute os desafios dos tratamento deste tipo de comorbidade, sugere que os serviços especializados num ou noutro transtorno precisam estar articulados, funcionando em rede e buscando criar estratégias que facilitem e garantam o acompanhamento integral dessas pessoas.

Até o ano de 2003, o Brasil não tinha uma política pública especifica para os problemas de saúde decorrente do uso prejudicial de álcool e outras drogas. ${ }^{19}$ A partir desta data, surge no contexto do SUS uma política com diretrizes claras, que propõe o acompanhamento preferencial destas pessoas em serviços comunitários, abertos, do tipo Centros de Atenção Psicossocial (CAPSs), em ações articuladas com atenção básica, ambulatórios especializados e leitos hospitalares em hospital geral. Em cidades entre 70 a 200 mil habitantes, CAPSs especializados na questão de álcool e outras drogas têm o encargo de dirigir a construção e articulação efetiva desta rede de assistência. No período de 2003 a 2010, 253 CAPSs AD foram criados. ${ }^{20}$ Após este esforço inicial, resta ainda enfrentar o enorme desafio de articulação dos serviços da rede para que o cuidado a essa clientela, que em geral sofre de diferentes tipos de comorbidades, seja de fato integral, longitudinal, e especialmente focado no enfrentamento dos problemas que decorrem do uso das substâncias psicoativas, e não exclusivamente centrada na interrupção do uso.

O objetivo deste artigo é indicar como se apresentou a frequência de sofrimento psíquico em pessoas sob tratamento em centros especializados em problemas relacionados ao uso prejudicial de álcool e outras drogas, na cidade de Macaé, localizada no norte do estado do Rio de Janeiro, ano de 2010, e identificar alguns aspectos do cuidado oferecido em tais centros, tanto para os problemas decorrentes do uso de substâncias psicoativas quanto para os problemas de saúde mental associados. Este estudo é parte de um estudo maior, multicêntrico, que envolve os países Panamá, Guatemala, Uruguai, Paraguai, Jamaica e Chile. No Brasil foram estudadas duas cidades: Macaé (206.748 habitantes) e Brasília (2,8 milhões de habitantes).

\section{MÉTODO}

Foi realizado um estudo epidemiológico transversal na população sob tratamento para problemas decorrentes do uso de álcool e drogas em todos os centros de assistência especializada da cidade de Macaé. Apesar das limitações dos estudos de corte seccional, este tipo de estudo 
é considerado o mais eficiente para se conhecer rapidamente uma realidade não investigada previamente, se obter uma avaliação breve da situação atual e também o mais factível sob condições de restrição orçamentária.

A prevalência de sofrimento psíquico na população de pacientes sob tratamento para problemas decorrentes do uso de álcool/drogas é a medida epidemiológica de frequência mais importante do presente estudo, embora outros dados relevantes tenham sido coletados. Um questionário, adaptado do formulário EULAC-CICAD, sob autorização prévia, foi utilizado. Trinta questões geraram informações sobre status socioeconômico e demográfico, história do abuso de álcool e drogas, história judicial, problemas mentais no passado e na atualidade, e alguns aspectos sobre o centro de tratamento. Avaliou-se ainda a Funcionalidade Familiar Percebida, com aplicação do instrumento APGAR-Famíliar. ${ }^{21-22}$ Nesta escala, que inclui cinco campos (adaptabilidade, parceria, crescimento, afeição e resolutividade), os valores possíveis vão de 0 a 10, baseados na satisfação do participante com as relações dentro de sua família. A interpretação do APGAR-Familiar é baseada no escore abaixo discriminado, sendo que cada questão tem valores de zero a dois. Um escore total de sete ou mais sugere uma percepção de alta funcionalidade familiar; entre quatro e seis, sugere moderada disfunção familiar, e três ou menos reflete grave disfunção familiar.

O sofrimento psíquico foi rastreado mediante a aplicação do Instrumento Kessler-10 (K-10), questionário de 10 itens que identifica a presença do Sofrimento Psíquico desde uma perspectiva orientada por sintomas. ${ }^{23-26}$ Os valores possíveis vão de 10 (indicando a ausência de sofrimento psíquico) até 50 (indicando grave sofrimento psíquico). A categorização do sofrimento psíquico se faz em quatro grupos: baixo (10-15), moderado (16-21), alto (22-29) e muito alto (30-50). Para propósitos analíticos, a maioria dos estudos une as duas últimas categorias (alto e muito alto). Um nível muito alto de sofrimento psíquico está associado com uma alta probabilidade da presença de transtornos de ansiedade ou depressão. ${ }^{23}$ Foram utilizadas versões validadas para português para o APGAR-Familiar e escala K-10. Utilizou-se o programa Epi Info versão 3.5.1 (CDC, 2008), de domínio público, para entrada e análise dos dados.

A coleta de informações e a observação do campo foi realizada pelo investigador principal e pelos investigadores assistentes composta por seis estudantes de graduação dos cursos de medicina e enfermagem da Universidade Federal do Rio de Janeiro/Campus Macaé, treinados em curso de capacitação de 40 horas pela investigadora principal e professora do curso de medicina da referida universidade, nos meses de abril a junho de 2010. Os questionários preenchidos e os termos de consentimento livre e esclarecido foram guardados em volumes separados a fim de garantir a segurança e confidencialidade dos mesmos.

Foram entrevistadas 60 pessoas em tratamento em centros especializados na cidade de Macaé. O tamanho da amostra foi calculado considerando a capacidade de detectar uma prevalência de $40,0 \%$ de sofrimento psíquico em níveis alto e muito alto, considerando um ponto de corte de 22 na escala K-10. ${ }^{28} \mathrm{~A}$ amostra foi dimensionada de modo a detectar uma diferença mínima de 5,0\% na prevalência, mantendo os critérios de erros tipo I e II nos níveis de 0,05 e 0,20, respectivamente. A população-alvo foi composta da totalidade de pessoas de ambos os sexos, maiores de 18 anos, que se encontra em tratamento em centros de tratamento públicos e privados, especializados em problemas decorrentes do uso de álcool e outras drogas. Foram excluídos os incapazes de responder ao questionário/entrevista devido a problemas mentais ou de inteligência que impedissem a comunicação ou compreensão, aqueles com comportamentos agressivos no momento da abordagem, com intoxicação, e/ou aqueles que recusassem a participação voluntária.

Foram acessados, para que a cobertura da população estimada fosse garantida, os três centros especializados para o tratamento dos transtornos decorrentes do uso de substâncias psicoativas existentes na cidade de Macaé: um centro de atenção diária, público, aberto, especializado no tratamento de pessoas com problemas decorrentes do uso prejudicial de álcool e outras drogas (CAPSs-AD) e dois centros de internação - um conveniado à rede pública de assistência em saúde e um privado - específicos para a mesma população.

O estudo foi aprovado pelo Comitê de Ética e Pesquisa do Centro de Adição e Saúde Mental do Canadá (CAMH), protocolo 225/2008, e pelo Comitê de Ética e Pesquisa da Escola de Enfermagem Anna Nery, da Universidade Federal do Rio de Janeiro, protocolo n ${ }^{\circ}$ 096/2009. A participação dos sujeitos da pesquisa foi voluntária e todos assinaram Termo de Consentimento Livre e Esclarecido. 


\section{RESULTADOS}

\section{Aspectos sociodemográficos}

Das 60 pessoas entrevistadas, $88,3 \%(n=53)$ são do sexo masculino, enquanto $11,7 \%(n=7)$ são do sexo feminino. Este dado é compatível com a característica dos centros estudados: apenas um dos serviços atende mulheres. Os demais centros são restritos a homens. Em relação à idade, a maioria delas, 31 das pessoas entrevistadas 51,5\% estão na faixa de 24 a 39 anos de idade, seguidos imediatamente pelas pessoas na faixa etária de mais de 40 anos, onde encontram-se 24 pessoas do total entrevistado, perfazendo $40,3 \%$ da mostra. Identificaram-se como solteiros 35 pessoas, perfazendo 59,3\% da amostra. Em relação à nacionalidade, a grande maioria é de brasileiros, 98,3\% $(n=59)$. Em relação à raça e grupo étnico, $60 \%(n=36)$ da mostra, se autoidentificou como parda (Tabela 1). Referiram viver em casa de suas famílias de origem, nos últimos 30 dias anteriores ao início deste tratamento, 38,3\% $(\mathrm{n}=23)$; em casa própria, da qual são proprietários, $35 \%(n=21)$; seguidos de $15 \%(n=9)$ que vivem em casa própria porém alugada. Disseram viver sozinhas 30\% ( $n=18)$, seguidos imediatamente por aqueles que disseram viver com a mãe, $28,3 \%(n=17)$, e por aqueles que vivem com esposo/esposa, $20 \%(n=12)$.

Tabela 1 - Distribuição das características sociodemográficas de pessoas em tratamento em centros especializados em problemas decorrentes do uso de álcool e outras drogas em Macaé-RJ, Brasil, 2010

\begin{tabular}{llrr}
\hline Variável & Categoria & $\mathbf{n}$ & \multicolumn{1}{c}{$\%$} \\
\hline \multirow{2}{*}{ Sexo } & Feminino & 7 & 11,7 \\
& Masculino & 53 & 88,3 \\
Idade & $18-23$ & 5 & 8,2 \\
& $24-39$ & 31 & 51,5 \\
& $40+$ & 24 & 40,3 \\
& Solteiro(a) & 35 & 59,3 \\
Estado civil & Casado(a) & 8 & 13,6 \\
& Divorciado(a) & 6 & 10,2 \\
& Separado(a) & 4 & 6,8 \\
& União estável & 5 & 8,5 \\
& Viúvo (a) & - & - \\
Nacionalidade & Não responderam & 1 & 1,7 \\
& Nacional & 59 & 98,3 \\
& Estrangeiro & 1 & 1,7 \\
& Branco & 18 & 30,0 \\
Raça/Grupo étnico & Pardo & 36 & 60,0 \\
& Preto & 6 & 10,0 \\
& Índio & - & - \\
& NR & - & - \\
\hline
\end{tabular}

Características educacionais e ocupacionais

Em relação às características educacionais foram investigadas as variáveis "sabe ler" e "sabe escrever" e nível educacional. Em relação à ocupação, foi estudada a variável "ocupação nos últimos 30 dias anteriores ao inicio de tratamento neste centro". Para esta variável foi permitido a resposta a mais de uma categoria. Em relação às características sócio-educacionais, a frequência indicou que a quase totalidade dos entrevistados sabia ler e escrever: $95 \%(n=57)$ sabe escrever, e 96,7\% ( $n=58)$, sabe ler. A grande maioria tinha nível educacional primário incompleto, $30 \%(n=18)$, seguido pelo mesmo número de $30 \% \quad(n=18)$ com nível secundário completo. Em relação à ocupação, 46,7\% (n=28) responderam estar empregados ou trabalhando como autônomo, enquanto 16,7\% ( $\mathrm{n}=10)$ disseram estar desempregados.

\section{Distribuição dos tipos de drogas utilizadas e tempo de tratamento recebido}

Foi investigada a frequência dos tipos de drogas referida pelos entrevistados como primeira droga de importância, utilizada nos últimos 30 dias, pela qual buscou o centro de tratamento especializado. A principal droga foi o álcool con $53 \%$ $(n=32)$. A cocaína veio a seguir tendo sido referida por 24 pessoas: sob a forma de pó foi citada por $25 \%(n=15)$ e sob a forma de crack foi citada por $15 \%(n=9)$. A maconha foi referida apenas por $5 \%$ $(n=3)$ como a principal droga pela qual se buscou tratamento. Não foram citados: hipnóticos e sedativos, estimulantes, opióides, abuso de medicação receitada por médicos, inalantes alucinógenos e esteróides anabolizantes.

A frequência dos tipos de drogas referida pelo entrevistado como segunda droga de importância, utilizada nos últimos 30 dias, pela qual buscou o centro de tratamento especializado repete a distribuição das drogas referidas como primeira droga. Foram citadas o álcool, a cocaína e a maconha, na seguinte ordem: o álcool con $34 \%(n=11)$; a cocaína também foi referida por 11 pessoas, sendo que $21,9 \%(n=7)$ a referiram sob forma de pó, e 12,5\% (n=4) a referiram sob forma de crack. A maconha con $21,9 \%(n=7)$. Nenhuma outra droga foi citada. Observa-se que $49 \%(n=28)$ do total de 60 referiu uma segunda droga como motivadora de busca de tratamento.

A terceira droga mais importante, utilizada nos últimos 30 dias antes do tratamento atual, 
que determinou a busca pelo tratamento, foi a maconha con $35,3 \%(n=6)$. A ela seguiu-se a cocaína quatro pessoas referiram o pó, com frequência de $23 \%$ para esta forma de apresentação da cocaína e uma pessoa o crack, determinando a frequência de $5,9 \%$ para esta última. O álcool, apresentou a menor frequência $17,6 \% \quad(n=3)$. Nenhuma outra droga foi referida. Do total de entrevistados, 14 referiram uma terceira droga como responsável pela busca de tratamento, ou seja, $25 \%$ da amostra.

\section{Encaminhamento aos centros de tratamento}

A principal forma de encaminhamento aos centros de tratamento foi a sugestão de amigos ou familiares, $40 \%(\mathrm{n}=24)$; seguida pela ida voluntária $28,3 \%(n=17)$; indicação feita por outro programa de tratamento de drogas $15 \%(n=9)$; por unidade de saúde primária $8,3 \%(n=5)$; unidade de saúde secundária e terciária e justiça ou polícia $5 \%(n=3)$; patrão ou empregador $5 \%(n=3)$; e serviços sociais $1,7 \%(n=1)$. Alguns tiveram mais de uma forma de encaminhamento.

\section{Condições referidas pelos pacientes, prévias ao tratamento nestes centros especializados, relacionadas ao diagnósticos de outros trans- tornos psiquiátricos, ao número de vezes em que foram tratados e ao tratamento recebido anteriormente}

A frequência dos diagnósticos psiquiátricos referidos pelos pacientes, prévio à entrada nos centros especializados no tratamento do uso prejudicial de álcool e outras drogas, foi constituída a partir de uma lista de cinco alternativas informadas e consultadas uma a uma. O diagnóstico mais frequente foi a depressão com $35 \%(n=21)$, imediatamente seguido de ansiedade com 33,3\% $(\mathrm{n}=20)$. Foram também referidos transtorno bipolar com 13,3\% ( $\mathrm{n}=8)$, e esquizofrenia com 1,7\% $(n=1)$. Observa-se que mais de um diagnóstico podia ser referido pela mesma pessoa.

Foi investigado o número de vezes em que algum tratamento prévio foi referido e o tipo de tratamento referido. A categoria "nenhum tratamento prévio" com 39,0\% ( $n=23)$, seguido pela categoria "mais de uma vez" com 32,3\% $(n=19)$ e pela categoria "uma vez" com $28,8 \%$ $(n=17)$. Apenas um informante não respondeu. Com relação ao tipo de atenção referido para o período prévio ao tratamento neste centro, o tratamento em unidade de internação especializada no tratamento para drogas alcançou a maior frequência com 35,\% (referido 21 vezes). Os grupos de autoajuda tiveram frequência de $26,7 \%$ (referidos 16 vezes). As consultas externas apresentaram a $3^{\mathrm{a}}$ maior frequência com $25 \%$, (referidos 15 vezes). Observar que com relação ao tipo de atenção referido, embora o " $\mathrm{n}$ " fosse de 60, um tipo de tratamento pôde ser referido mais de uma vez.

\section{Condições atuais dos pacientes nos centros de tratamento}

\section{Frequência de outros diagnósticos referidos durante o tratamento nestes centros}

A frequência dos diagnósticos referidos que não são diretamente relacionados ao uso de drogas, durante o tratamento atual nos centros de tratamento especializados, foi de 38,3\% (referido 23 vezes) para ansiedades, seguido de depressão, com frequência de 28,3\% (referido 17 vezes) e transtorno bipolar, com frequência de 15\% (referido nove vezes). Observar que mais de um diagnóstico poderia ser referido por um mesmo informante.

\section{Frequência do tratamento referido para cada uma das outras condições diagnósticas nestes centros}

A frequência dos tratamentos referidos para cada um das condições diagnósticas identificadas numa lista de cinco alternativas, perguntadas uma a uma foi de 95,7 (referido 22 vezes) para a ansiedade, sugerindo que nem todos que a referiram receberam tratamento para a condição patológica citada. O mesmo se passou com a depressão e com a esquizofrenia, que apresentaram frequências de $94,1 \%$ e $50 \%$ respectivamente. Apenas o transtorno bipolar alcançou frequência de $100 \%$, ou seja, o tratamento foi referidos para todos que citaram o transtorno.

\section{Frequência do tipo de tratamento referido para cada uma destas outras condições diagnósticas nestes centros}

Para o levantamento da frequência com que diferentes tipos de tratamento foram referidos como modalidades de tratamento oferecido 
nos centros especializados, foi apresentado ao informante uma lista de 10 tipos de tratamento e perguntado se cada um deles lhe era oferecido ou não. A terapia de grupo alcançou a principal frequência $90 \%(n=54)$, seguido do aconselhamento com $68,3 \%(n=41)$, da psicoterapia com $61,7 \%$ $(n=37)$, do grupo de apoio com 51,7\% ( $n=31)$, da auto-ajuda com $46,7 \%(n=28)$, da terapia familiar com $45 \%(n=27)$, medicação com $40 \%(n=24)$ e da meditação com $36,7 \%(n=22)$. O único tipo de tratamento, dos listados, que não foi referido foi a yoga. Cada tipo de tratamento pôde ser citado por mais de um informante.

\section{Frequência da satisfação com o tratamento atual}

Do total da amostra 98,3\% $(n=59)$ pessoas responderam estar satisfeitas como o tratamento recebido, somente $1,7 \%(n=1)$ dos informantes respondeu estar insatisfeito.
Frequência do sofrimento psíquico e percepção da funcionalidade familiar

A distribuição do sofrimento psíquico na população estudada, de acordo com as categorias proposta pela escala de Kessler-K10 foi a seguinte (Tabela 2): as categorias "muito severo" e "severo" obtiveram as principais frequências 34,0\% $(n=20)$ e $27,2 \%(n=16)$, respectivamente, imediatamente seguidos pela categoria "moderado", que alcançou frequência de 22,1\% ( $n=13)$. A categoria "baixo" sofrimento psíquico teve uma frequência de $17 \%(n=10)$.

Em relação à Funcionalidade Familiar Percebida (Tabela 2), a distribuição da categoria "normofuncional" foi a principal entre os pacientes em tratamento nos centros especializados, alcançando a frequência de 65,5\% (n=38). As categorias de "moderadamente disfuncional" e "severamente disfuncional" alcançaram frequência de 18,9\% $(n=11)$ e $15,5 \%(n=9)$, respectivamente.

Tabela 2 - Frequência de sofrimento psiquíco e Funcionalidade Familiar Percebida entre pessoas sob tratamento em centros especializados em problemas decorrentes do uso de álcool e outras drogas em Macaé-RJ, Brasil, 2010

\begin{tabular}{llcc}
\hline Variável & Categoría & Sim & \% \\
\hline & Baixo (10-15) & 10 & 17 \\
Sofrimento psíquico (escala K-10) & Moderado (16-21) & 13 & 22 \\
& Severo (22-29) & 16 & 27 \\
& Muito severo (30-50) & 20 & 34 \\
\hline Total & & $\mathbf{5 9}$ & $\mathbf{1 0 0}$ \\
\hline \multirow{2}{*}{ Funcionalidade familiar percebida (APGAR-família) } & Normofuncional (>7) & 38 & 65,5 \\
& Moderadamente disfuncional(4-6) & 11 & 19,0 \\
& Severamente disfuncional $(<3)$ & 9 & 15,5 \\
\hline Total & & $\mathbf{5 8}$ & $\mathbf{1 0 0}$ \\
\hline
\end{tabular}

\section{DISCUSSÃO}

A prevalência de sofrimento psíquico na população de pessoas sob tratamento para uso prejudicial de álcool/drogas foi considerada a medida epidemiológica de frequência mais importante no presente estudo. Foi a partir do levantamento da sua distribuição que se buscou identificar a provável presença da comorbidade entre outros transtornos mentais e transtorno decorrente do uso prejudicial de álcool e outras drogas.

A identificação da presença de sofrimento psíquico nesta população específica, a partir da escala de Kessler K-10, permite responder à seguinte pergunta: existe evidência de possível problema mental nesta população específica que requeira maior investigação? Os dados encontrados nos permitem responder que sim. Do total de 60 pessoas entrevistas $60 \%(n=36)$, apresentam sofrimento psíquico no grau severo e muito severo, indicando o alto risco desta população para ansiedade e depressão, em especial.

As outras variáveis ou possíveis fatores associados ao problema da comorbidade entre os problemas decorrentes do uso prejudicial de álcool e outras drogas e os outros transtornos psiquiátricos, levantados neste estudo, nos indicam as características da população estudada. Em relação aos aspectos sociodemográficos temos uma população de maioria de homens com $88,3 \%$ dos entrevistados. Embora seja sabido que o problema de álcool e outras drogas é mais comum entre pessoas do sexo masculino, a proporcionalidade dos 
dados relacionados ao sexo está marcada pelo fato de apenas uma das três instituições investigadas oferecer atenção às mulheres. Em relação à idade, a maioria das pessoas entrevistadas (51,5\%), está na faixa de 24 a 39 anos de idade, seguidos imediatamente pelas pessoas na faixa etária de mais de 40 anos, que constitui $40,3 \%$ do total da mostra. Esses dados não correspondem proporcionalmente ao que é observado no restante do país, onde a faixa de pessoas de 18 a 24 , segundo dados gerais para o país, ${ }^{15}$ indicam que o problema da dependência na faixa etária de 18 a 23 é proporcionalmente maior do que encontrado nas instituições estudadas, onde apenas $8 \%$ da população nesta faixa etária apresentava este problema, ao contrário dos dados do país, em que aproximadamente $23 \%$ de pessoas nesta faixa etária apresenta problema de dependência. Em relação à nacionalidade, a grande maioria da mostra é de brasileiros (98,3\%) $(n=59)$, embora dados do Censo de 2005 indiquem que $10 \%$ da população da cidade de Macaé seja atualmente constituída por estrangeiros. Em relação à raça e grupo étnico, $60 \%$ da mostra se autoidentificou como parda. Viveram nos últimos 30 dias em casa de sua família de origem 38,3\% dos entrevistados, $35 \%$ em casa própria em que é o proprietário do imóvel, seguidos por $15 \%$ que vivem em casa própria, porém alugada. A grande maioria dos entrevistados disse viver só em 30\% dos entrevistados. Vivem com a mãe $28,3 \%$ e com esposo/esposa $20 \%$ dos entrevistados.

Em relação à escolaridade, $95 \%$ da amostra sabia ler e 96,7\% sabe escrever. O nível educacional primário incompleto e secundário incompleto, responde por $60 \%$ da amostra. Do total de pessoas entrevistadas, $46,7 \%$ se diz empregado ou trabalhando como autônomo, enquanto $16,7 \%$ se diz desempregado.

A principal droga pela qual se buscou tratamento foi o álcool, referida por $53 \%$ da amostra. A cocaína em pó, referida por $25 \%$ dos entrevistados, veio em $2^{\circ}$ lugar, seguida pelo crack, referido por $15 \%$ dos entrevistados. Estes dados indicam, repetindo o padrão epidemiológico dos estudos brasileiros, que o uso prejudicial de álcool continua a ocupar o lugar de principal problema neste campo. A principal forma de encaminhamento aos centros de tratamento foi a sugestão de amigos ou familiares, citada por $40 \%$ dos entrevistados.

O diagnóstico prévio referido mais frequente foi a depressão, citado por $35 \%$ dos informantes, imediatamente seguido de ansiedade, citado por $33,3 \%$. A ansiedade foi o principal diagnóstico atual referido, com frequência de $38,3 \%$, seguido de depressão, com frequência de 28,3\%. A funcionalidade familiar foi percebida como normal para $65,5 \%$ da amostra. Embora este dado tenha sido inesperado, ${ }^{28-30}$ ele se repetiu em outros sítios investigados neste estudo multicêntrico, exigindo mais estudo para a compreensão global de sua determinação.

\section{CONCLUSÕES}

A frequência da categoria sofrimento psíquico entre a população estudada, avaliada a partir da aplicação da escala de Kessler-K10, foi eficaz para identificar o risco de transtorno mental neste grupo específico. Os dados encontrados corroboram o que é visto na literatura internacional, ou seja, o alto risco de esta população, no caso do presente estudo $60 \%$ dela, apresentar sofrimento psíquico no grau severo ou muito severo, o que significa igualmente alta probabilidade da presença de transtorno tipo ansiedade e depressão, principalmente.

A presença de transtorno mental referido durante o tratamento atual para os transtornos de depressão e ansiedade, quadros mais comuns neste grupo específico, foi de 38,3\% para ansiedade e de $28,3 \%$ para depressão. Ressalta-se assim a importância de tomar a questão da associação não apenas de mais de um diagnóstico, como também a associação de sintomas psiquiátricos que não chegam a constituir diagnósticos psiquiátricos estrito senso, como problema relevante dos desenhos tanto das redes de assistência e dos serviços, quanto das ações que prestam cuidado a essa clientela.

\section{Limitações}

As limitações desta pesquisa relacionam-se especialmente a três aspectos: ao tipo de estudo, à seleção dos locais de estudo e ao tipo de questionário utilizado. Em relação ao tipo de estudo, é provável que haja vieses de prevalência, devido ao fato de que, em um estudo de corte seccional, apenas são coletados dados de um momento na vida dos participantes. Como consequência, perde-se a visão mais dinâmica dos eventos de vida, tais como migração, morte e incidência de outras doenças. A falta de acompanhamento dos participantes impede uma percepção clara da sequência dos eventos (tanto de exposições como de outras variáveis de efeito), limitando inferências de causa-efeito. Uma outra limitação 
relaciona-se à seleção dos locais de estudo, cujas características podem variar de acordo com a estrutura e organização dos serviços de saúde. Portanto, as diversas situações de acesso aos serviços e suas características limitam a capacidade de generalizar os resultados para a população-alvo em uso prejudicial de álcool e outras drogas. $\mathrm{O}$ uso de um questionário auto-administrado como meio de coleta de dados também constituiu uma limitação. Este efeito foi minimizado pela ajuda proporcionada pelos assistentes de pesquisa, mas não foi excluído.

\section{Recomendações}

Sensibilizar os profissionais dos diferentes centros para tratamento dos problemas decorrentes do uso do álcool e das outras drogas da cidade em relação à alta prevalência de sofrimento psíquico nesta clientela.

Capacitar os profissionais dos diferentes centros para tratamento dos problemas decorrentes do álcool e outras drogas da cidade para identificarem o sofrimento psíquico em seus diferentes graus.

Sensibilizar e capacitar os profissionais dos diferentes centros para tratamento dos problemas decorrentes de álcool e outras drogas da cidade a trabalharem articulados e em rede com os serviços da rede de saúde local especializados no cuidado a pessoas com problemas psiquiátricos, especialmente atenção básica e CAPS.

Sensibilizar os profissionais da atenção básica para a alta prevalência da associação de problemas decorrentes do uso de álcool e outras drogas e sofrimento psíquico e capacitá-los para identificar o problema.

Capacitar os profissionais da atenção básica a trabalharem articulados e em rede com os serviços especializados no cuidado a pessoas com problemas decorrentes de álcool e outras drogas e com problemas psiquiátricos, para que possam assistir adequadamente os que apresentem esta comorbidade.

\section{AGRADECIMENTOS}

Este estudo contou com o apoio, assessoria e patrocínio do governo do Canadá, da Organização dos Estados Americanos, da Comissão Interamericana para o Controle do Abuso de Drogas e do Centro de Adição e Saúde Mental do Canadá, além do apoio da Fundação Educacional de Macaé, que financiou cinco bolsas de auxiliares de pesquisa, por um ano, para alunos de graduação dos cursos de Medicina e Enfermagem da Universidade Federal do Rio de Janeiro-Campus Macaé. Agradeço também à Coordenação de Saúde Mental do Município de Macaé, e à coordenação do CAPS AD, bem como aos coordenadores dos demais Centros investigados e aos seus profissionais pelo interesse em garantir a plena realização do estudo. A participação dos alunos de graduação de Enfermagem e Medicina da UFRJ-Campus Macaé foi imprescindível para o desenvolvimento da pesquisa, bem como dos pacientes que gentilmente aceitaram responder aos questionários, sem os quais este estudo não teria acontecido.

\section{REFERÊNCIAS}

1. Colin C. Substance abuse issues and public policy in Canada. Canada's federal drug Strategy. 2006 [acesso 2009 Jun 25]. Disponível em: www.parl. gc.ca/information/library/PRBpubs//prb0615-e. html

2. Conway KP, Compton W, Stinson FS, Grant BF. Lifetime comorbidity of DSM-IV mood and anxiety disorders and specific drug use disorders: results from the National Epidemiologic Survey on alcohol and related conditions. J Clin Psychiatry. 2006 Feb; 67(2):247-57.

3. Drake R, McLaughlin P, Pepper B, Minkoff K. Dual diagnosis of major mental illness and substance disorder: an overview. New Dir Ment Health Serv. 1991 Summer; (50):3-12.

4. Hersh DF, Modesto-Lowe V. Drug abuse and mood disorders. In: Henry BK, Bruce J, Rounsaville MD, editors. Dual diagnosis and treatment. New York (US): Marcel Dekker Inc.; 1998. p. 177-201.

5. Kranzler HR, Mason B, Modesto-Lowe V. Prevalence, diagnosis, and treatment of comorbid mood disorders and alcoholism. In: Kranzler HR, Rounsaville B, editors. Dual diagnosis and treatment. New York (US): Marcel Dekker Inc.; 1998. p. 107-36.

6. Brady S, Hiam CM, Saemann R, Humbert L, Fleming M, Dawkins-Brickhouse K. Dual diagnosis: a treatment model for substance abuse and major mental illness. Community Ment Health J. 1996 Dec; 32(6):573-8.

7. Dalex DC, Moss HB. Dual disorders. counseling clients with chemical dependency and mental illness. $3^{\mathrm{a}}$ ed. Minnesota (US): Hazelden Foundation Center; 2002.

8. Drake R, Mueser KT, Brunette M, McHugo GJ. A review of treatments for people with severe mental illnesses and co-occurring substance use disorders. Psychiatr Rehabil J. 2004 Spr; 27(4):360-74.

9. Drake R, Mueser K, Clark R, Wallach M. The course, treatment, and outcome of substance 
disorder in persons with severe mental illness. Am J Orthopsychiatry. 1996 Jan; 66(1):42-51.

10. Evans K, Sullivan JM. Dual diagnosis. counseling the mentally III substance abuser. New York (US): The Guilford Press; 2001.

11. Grant BF. The influence of comorbid major depression and substance use disorders on alcohol and drug treatment: results of a national survey. In: National Institute on Drug Abuse (NIDA). Treatment of drug-dependent individuals with comorbid mental disorders. Rockville (US): NIH; 1997.

12. Fortes S. Transtornos mentais comuns na atenção primária: suas formas de apresentação, perfil nosológico e fatores associados em unidades do programa de saúde da família do município de Petrópolis, Rio de Janeiro [tese]. Rio de Janeiro (RJ): Universidade do Estado do Rio de Janeiro, Instituto de Medicina Social; Rio de Janeiro; 2004.

13. Tanaka OY, Ribeiro L. Desafio para a atenção básica da assistência em saúde mental. Cad Saúde Pública. 2006 Set; 22(9):1845-53.

14. Saraceno B, Saxena S, Caraveo-Adnurga J, Khon R, Levav I, Caldas de Almeida M. Los trastornos mentales en América Latina y el Caribe: asunto prioritario para la salud publica. Rev Panam Salud Publica. 2005 Nov; 18(4-5):229-40.

15. Carlini, EA, Galduróz JCF, Noto AR, Nappo SA. I Levantamento domiciliar sobre o uso de drogas psicotrópicas no Brasil: estudo envolvendo as 107 maiores cidades do país: 2001. São Paulo: Universidade Federal de São Paulo, Centro Brasileiro de Informações Sobre Drogas Psicotrópicas; 2002.

16. Carlini EA, Galduróz JC, Noto AR, Carlini CM, Oliveira LG, Nappo AS, et al. II Levantamento domiciliar sobre o uso de drogas psicotrópicas no Brasil: estudo envolvendo as 107 maiores cidades do país: 2005. São Paulo: Universidade Federal de São Paulo, Centro Brasileiro de Informações Sobre Drogas Psicotrópicas; 2007.

17. Mari J, Jorge MR, Kohn R. Epidemiologia dos transtornos psiquiátricos em adultos. In: Mello MF, Mello AAF, Kohn R, organizadores. Epidemiologia da saúde mental no Brasil. Porto Alegre (RS): Artmed; 2007. p.119-41.

18. Almeida-Filho N, Mari JJ, Coutinho ESF, Franca JF, Fernandes J, Andreoli SB, et al. Brazilian multicentric study of psychiatric morbidity :methodological features and prevalence estimates. Br J Psychiatry. 1997 Dec; 171:524-9.

19. Delgado PGG, Schechtman A, Weber R, Amstalden AF, Bonavigo E, Cordeiro F, et al. Reforma psiquiátrica e política de saúde mental no Brasil.
In: Mello MF, Mello AAF, Kohn R, organizadores. Epidemiologia da saúde mental no Brasil. Porto Alegre (RS): Artmed; 2007. p. 39-83.

20. Ministério a Saúde (BR). Saúde mental em dados V. ano III, $\mathrm{n}^{\circ}$ 5. Informativo eletrônico. Brasília (DF): Coordenação Geral de Saúde Mental, Álcool e Outras Drogas; 2008 [acesso 2011 Jan]. Disponível em: www.saude.gov.br/saudemental.

21. Smilkstein G. The Family APGAR: a proposal for family function test and its use by physicians. J Fam Prac.1978 Jun; 6(6):1231-9.

22. Smilkstein G, Ashworth C, Montano D. Validity and reliability of the family APGAR as a test of family function. J Fam Prac. 1982 Aug; 15(2):303-11.

23. Andrews G, Slade T. Interpreting scores on the Kessler Psychological Distress Scale (K10). Aust N Z J Public Health. 2001 Dec; 25(6):494-7.

24. Australian Bureau of Statics. Use of the Kessler Psychological Distress Scale in ABS health and wellbeing survey, psychological distress in the Western Australian population Australia, 1997 and 2001 [online].[acesso 2009 Jun 25] Disponível em: www.abs.gov.au/ausstats/abs@. nsf/.../4817.0.55.001

25. Kessler RC, Andrews G, Colpe LJ, Hiripi E, Mroczek DK, Normand SLT, et al. Short screening scales to monitor population prevalances and trends in nonspecific psychological distress. Psychol Med. 2002 Aug; 32(6):959-76.

26. Pratt L, Dey A, Cohen A. Characteristics of adults with serious psychological distress as measured by the K6 Scale: United States, 2001-04. Adv Data. 2007 Mar 30; (382):1-18.

27. Van Ours JC, Willians J. Cannabis use and mental health problems. Discussion Papers. 2009 Jul; (60):238.

28. Clark RE. Family support and substance use outcomes for persons with mental illness and substance use disorders. Schizophr Bull. 2001; 27(1):93-101.

29. Dixon L, McNary S, Lehman A. Substance abuse and family relationships of persons with severe mental illness. Am J Psychiatry. 1995 Mar; 152(3):456-8.

30. Rodríguez Martinez A, Pinzon Pulido SA, Máiquez Pereza, Herrera Jaimez J, DeBento Tornente Cuesta Orhz E. ¿Tienen apoyo social y familiar los drogodependientes que participan en el programa «libre de drogas» en prisión? Medicina de Familia [online]. 2004 Feb [acesso 2009 Nov 15]; 5(1). Disponível em: http://www.samfyc.es/Revista/ PDF/v5n1/04.pdf 